\title{
Suppression of the Lower Charge State Ions in the External Accumulation RF Multipole with a Reduced Trapping DC Potential
}

\author{
Aleksey V. Tolmachev, Andrey N. Vilkov, Ljiljana Paša-Tolic, \\ Harold R. Udseth, and Richard D. Smith \\ Environmental Molecular Science Laboratory, Pacific National Laboratory, Richland, Washington, USA
}

\begin{abstract}
Radio frequency (RF) multipoles are increasingly used in mass spectrometry as twodimensional ion traps for ion accumulation and preselection. It was reported recently that ions having lower charge states, in particular singly charged ions, can be efficiently removed from such an ion trap when reduced DC trapping voltages are applied. This procedure can be useful for removing singly charged species contributing chemical noise to mass spectra of complex biomolecular samples, e.g., solvent contaminants in LC-MS or relatively low MW ampholytes in CIEF-MS experiments. We consider a physical mechanism and derive relationships that provide a quantitative description for the low charge state ejection phenomenon. Experimental conditions for the efficient discrimination against lower charge states are evaluated. Initial experimental observations reported are in agreement with the theoretical treatment. (J Am Soc Mass Spectrom 2003, 14, 1229-1235) (c) 2003 American Society for Mass Spectrometry
\end{abstract}

$\mathrm{I}$ $\mathrm{t}$ is well known that ion optical devices themselves cannot separate ions having the same $\mathrm{m} / \mathrm{z}$ ratio but different charge states [1]. However, when collisions with neutrals are involved, the absolute values of the ion mass and charge may be of importance [2-7]. Here we consider a configuration that directly utilizes the ability of collisional multipoles to distinguish between different charge states, regardless of $\mathrm{m} / \mathrm{z}$ ratio.

Radio frequency (RF) collisional multipoles [3] are increasingly used in mass spectrometry as two-dimensional (2D) ion traps for ion accumulation and/or pre-selection [8-12]. It was reported recently that ions having lower charge states, in particular 1+ ions, can be efficiently removed from such an ion trap when reduced DC trapping voltages are applied [13-15]. Below we consider a possible mechanism for such a phenomenon and report on its evaluation in the context of initial experimental results.

\section{Theory}

The schematic of the collisional cooling RF multipole 2D ion trap is shown in Figure 1. It is assumed that the RF multipole contains a neutral bath gas, and that outside of the RF multipole the mean free path of ions is large compared to characteristic dimensions of electrodes, $\sim 1$ $\mathrm{cm}$. During the ion accumulation event, the DC poten-

Published online August 30, 2003

Address reprint requests to Dr. R. D. Smith, Environmental and Molecular Sciences Laboratory, Pacific Northwest National Laboratory, MS K8-98, P.O. Box 999, Richland, WA 99352, USA.

tial of the first orifice (1) is reduced to let ions inside the multipole. The DC potential at the exit orifice (2) is maintained at a positive voltage sufficient to trap the ions. The effective potential created by the multipolar RF field confines ions in the radial direction [16]. Collisions with the bath gas serve to dissipate initial kinetic energy of ions. The translational energy of ions drops exponentially down to the thermal level. The characteristic exponential time of the ion velocity relaxation is [4]:

$$
\tau=\frac{3\left(m+m_{g}\right)}{4 m_{g} n_{g} \sigma u_{k T}} ; u_{k T}=\sqrt{\frac{8 k T}{\pi m_{r}}}
$$

Here $m$ is the ion mass, $m_{g}$ is the bath gas molecular mass, $\sigma$ is the cross section of ion-molecule collision, $n_{g}$ is the number density of the bath gas, $k$ is the Boltzmann constant, $T$ is the temperature of the bath gas and $m_{r}=$ $m_{g} m /\left(m_{g}+m\right)$. The collisional relaxation has a characteristic time scale roughly similar for different ions, defined primarily by the bath gas pressure $p$ [4]. For the pressure range of interest here, 0.01 to $\sim 10^{-5}$ torr, the collisional relaxation time $\tau$ ranges from $\sim 0.1$ to $100 \mathrm{~ms}$.

Upon completion of the ion accumulation event, the DC potential at the entrance of the multipole is increased to create the trapping barrier. The ions can then be stored inside the trap during a considerable time, $>1$ s. Consider an ensemble of ions trapped in an RF multipole 2D ion trap after reaching thermal equilibrium with the bath gas. The kinetic energy of the thermalized ions acquires the Maxwell distribution [4, 

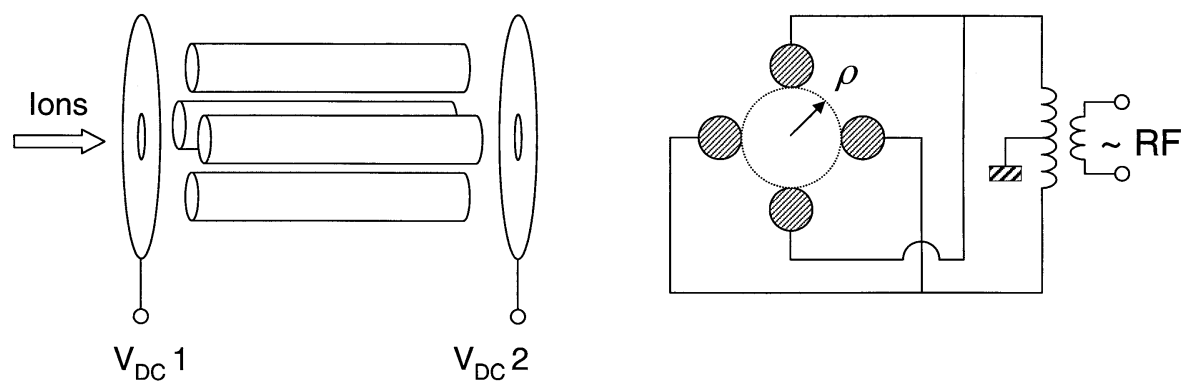

Figure 1. A diagram of the RF multipole 2D ion trap, the case of a quadrupole is shown. DC offsets $V_{D C} 1$ and $V_{D C} 2$ are applied to the input and exit orifices; the rods are DC grounded. The RF connection scheme is depicted on the cross section right view.

7]. For the ion velocity component along the multipole axis $v_{z}$ the distribution is as follows:

$$
F_{M}\left(v_{z}\right)=\frac{1}{\sqrt{\pi} v_{k T}} \exp \left(-\frac{v_{z}^{2}}{v_{k T}^{2}}\right)
$$

Here $v_{k T}$ is the thermal velocity for ion mass $m$ :

$$
v_{k T}=\sqrt{\frac{2 k T}{m}}
$$

The most energetic ions, corresponding to the so called high energy tail of the Maxwell distribution (2), may have a sufficient kinetic energy $K$ to surmount the DC trapping barrier:

$$
K=\frac{m v_{z}^{2}}{2}<z e U_{\text {trap }}
$$

Here the ion charge $z e$ is expressed as a number $z$ of elementary charges $e$. It follows that lower charge states can overcome the DC trapping potential $U_{\text {trap }}$ with lower axial kinetic energy. Substituting the energy in the distribution eq 2 we obtain $F_{M} \propto \exp (-K / k T)$. The fraction of ion population residing in the high energy tail of the thermal distribution increases exponentially with reducing the cut off energy $K_{0}=z e U_{\text {trap }}$. Thus, certain conditions provide strong discrimination between different charge states.

Approximately, all thermalized ions have on average a kinetic energy $\sim k T$, corresponding to the thermal energy of the bath gas molecules. This energy is the same for all ion species, independent of mass and charge. To overcome a DC barrier the ion kinetic energy must be higher than the barrier in Volts, multiplied by the ion charge. Thus, doubly charged ions experience a barrier, double that of singly charged ions.

We now proceed to obtain a quantitative relationship for the ion decay rate. Consider a quasi-equilibrium case, when the rate of the ion loss through the DC barrier is low enough so that the ion velocities closely mirror a Maxwell distribution, eq 2. In order to fulfill this condition the characteristic time for ion loss must be larger than the collisional relaxation time $\tau$, eq 1 . We also consider the limit of a low linear ion density, and neglect all the space charge interactions. Thus, the trapped ion species do not interact with each other, and we can consider each ion separately. Under such conditions the thermalized ions create a narrow cylindrical ion cloud having the length $L$, roughly equal to the multipole length. The ion cloud radius is defined by the strength of the effective potential applied [4, 7]. We assume that this radius is smaller than the exit orifice radius, which is typically the case under experimental conditions optimized for high ion transmission efficiency. Under the low space charge conditions, the number density of ions $n_{i}$ is maximal at the axis [7]. We can estimate the total number of trapped ions as $N_{\text {ions }}=$ $n_{i} L A$, where $A$ is the effective cross section area of the ion cloud.

Ions having velocity $v_{z}$ above the threshold eq 4 contribute to the flux of exiting ions as $d n_{i} v_{z} A$, where $d n_{i}$ $=n_{i} F_{m}\left(v_{z}\right) d v_{z}$ is the ions number density fraction corresponding to $v_{z}$. The cut off velocity is $v_{0}=\left(2 K_{0} / m\right)^{1 / 2}=$ $\left(2 z e U_{\text {trap }} / m\right)^{1 / 2}$. The total flux can be calculated as follows:

$$
\begin{aligned}
F_{\text {exit }} & =\int_{v_{0}}^{\infty} n_{i} F_{M}\left(v_{z}\right) A v_{z} d v_{z} \\
& =\frac{n_{i} A v_{k T}}{2 \sqrt{\pi}} \exp \left(-\frac{z e U_{\text {trap }}}{k T}\right)
\end{aligned}
$$

We see that the rate of ion loss is proportional to the total number of ions, which means that the ion decay is exponential:

$$
N_{\text {ions }}(t)=n_{i}(t) L A=N_{0} \exp \left(-t / \tau_{i}\right)
$$

Here $N_{0}$ is the initial number of trapped ions. The characteristic exponential time for the ion loss $\tau_{i}$ can be expressed as follows:

$$
\tau_{i}=\frac{N_{\text {ions }}}{F_{\text {exit }}}=\tau_{0} \exp \left(\frac{z e U_{\text {trap }}}{k T}\right), \tau_{0}=\frac{2 \sqrt{\pi} L}{v_{k T}}
$$


Table 1. Characteristic times $\tau_{0}$ and $\tau_{i}$, in seconds, calculated using eq 7 for $\mathrm{U}_{\text {trap }}=0.1 \mathrm{~V}, \mathrm{~T}=293 \mathrm{~K}$ and $\mathrm{L}=0.1 \mathrm{~m}$, for charge states $1+, 2+$, and $3+$

\begin{tabular}{lcccc}
\hline & $\tau_{0}$ for $m / z=400$ & $\tau_{0}$ for $m / z=2000$ & $\tau_{i}$ for $m / z=400$ & $\tau_{i}$ for $m / z=2000$ \\
\hline \hline$z=1$ & 0.0032 & 0.0072 & 0.169 & 0.378 \\
$z=2$ & 0.0046 & 0.0102 & 12.6 & 28.1 \\
$z=3$ & 0.0056 & 0.0125 & 807.3 & 1805 \\
\hline
\end{tabular}

We conclude that the characteristic time of decay depends exponentially on the charge state $z$. Lower charge states have exponentially lower decay rates, resulting in much shorter decay times.

An implication of the relationships 6 and 7 is that the ion decay is independent of the bath gas parameters, including pressure, molecular mass, and collision cross section. The primary relevant parameter is the ratio of DC barrier to thermal energy. The pre-exponent $\tau_{0}$ depends on the quadrupole length $L$ and thermal velocity of ions $v_{k T}$, eq 3 . Thus, the decay rate can be influenced not only by the ion charge ze, but also by its mass $m$. The dependence on $m$ becomes significant when the exponent in eq 7 is not large, i.e., when $z e U_{\text {trap }}$ $\sim k T$.

Since thermal energy is $k T \sim 0.025 \mathrm{eV}$, the $\mathrm{DC}$ barrier must be $U_{\text {trap }} \gg 25 \mathrm{mV}$ in order to obtain an efficient charge states separation. On the other hand, if the barrier is too high, the characteristic decay time $\tau_{i}$ becomes impractically long. As an example we consider the case of $U_{\text {trap }}=0.1 \mathrm{~V}$. We assume that the trapped ions have $\mathrm{m} / \mathrm{z}$ range from 400 to 2000 and charge states $1+, 2+$, and $3+$. Corresponding characteristic times $\tau_{0}$ and $\tau_{i}$ are listed in Table 1 . Using the exponential decay relationship 6 for $\tau_{i}$ values shown, one can estimate the ion abundances versus time. It can be seen that all charge states are preserved during a time $<0.1 \mathrm{~s}$. After $\sim 1$ s most of singly charged ions escape the DC barrier, while $2+$ and $3+$ maintain more than $90 \%$ of the initial abundance. Finally, a time delay of $\sim 100 \mathrm{~s}$ results in removing most of the $1+$ and $2+$ ions, while most of the $3+$ ions are still inside the trap.

We conclude that the efficient separation of lower charge state ions can be achieved using the approach described. In order to maintain quasi-equilibrium conditions, $\tau_{i}>\tau$, eq 1 , the bath gas pressure $p$ needs to be high enough, e.g., for $\tau_{i} \sim 100 \mathrm{~ms}: p>\sim 10^{-5}$ torr. A fine adjustment of the trapping voltage is required to achieve the separation. The voltage $U_{\text {trap }}=0.1 \mathrm{~V}$ considered in the above example is the potential barrier experienced by the trapped ions, which can be different from the actual DC voltage applied to the exit orifice. The potential barrier is a linear combination of the DC potential created by the orifice and the potential resulting from the DC offset applied to the ion extraction optics that follow the orifice. Generally, one can expect that the barrier $U_{\text {trap }}$ is slightly lower than that provided by the orifice voltage $V_{O R}$. It follows that in order to create $U_{\text {trap }}=0.1 \mathrm{~V}$, a somewhat larger potential must be applied to the orifice. To define exactly the depen- dence $V_{O R}$ versus $U_{\text {trap }}$ potential calculations should be carried out, taking into account the dimensions and potentials of the extraction ion optics.

Additionally, the space charge potential created by trapped ions also will contribute to the exit barrier. The contribution $V_{S C}$ can be significant, even for low trapped charge. The potential created by trapped ions at the exit of RF multipole has been roughly estimated in [5]:

$$
V_{S C}=z e n_{i} R_{q} \rho / 2 \varepsilon_{0}
$$

Here $R_{q}$ is the ion cloud radius, $\rho$ is the inscribed radius of the multipole, and $\varepsilon_{0}$ is the dielectric permittivity constant. Using eq 8 we can estimate the total number of trapped ions that results in a small enough perturbation of the trapping barrier, $V_{S C}<\sim k T / e$. For $z=2, R_{q}$ $=1 \mathrm{~mm}, \rho=4 \mathrm{~mm}$ and $L=10 \mathrm{~cm}$ we obtain:

$$
N_{0}<\frac{2 \pi \varepsilon_{0} k T R_{q} L}{z e^{2} \rho} \approx 10^{5}
$$

If the initial number of ions is larger than this, one may expect a rapid initial drop of all charge states, until a low level of the space charge potential is reached, $V_{S C} \ll$ $U_{\text {trap. }}$. This will degrade the charge state separation efficiency.

A possible way to maintain the charge separation for a larger number of trapped ions than the limit of eq 9 is to use increased trapping voltages, to account for $V_{S C}$ input. The ion charge decrease during the separation process will require a gradual decrease of the trapping potential.

If a low stationary DC barrier $U_{\text {trap }} \sim 0.1 \mathrm{~V}$ is used for charge states separation, then the trapped ion population must be controlled to be below the limit estimated in eq 9. One can increase the trapped ion charge wile maintaining low enough space charge potential, $V_{S C} \ll 0.1 \mathrm{~V}$, by using a higher order RF multipole as a 2D ion trap. As seen from eq 9 , the limit $N_{0}$ is proportional to $R_{q} / \rho$ ratio. We have used $R_{q} / \rho=0.25$, corresponding to an efficient storage of ions in the RF quadrupole $2 \mathrm{D}$ ion trap. It can be estimated that $\mathrm{RF}$ multipoles can store ions with $R_{q} / \rho$ ratio approaching 1 as the number of rods increases [5-7]. It follows that the charge separation using a multipole (e.g., octopole) will allow use of a larger number of trapped ions than a quadrupole. 


\section{Experiment}

In order to evaluate the above theoretical results we have performed experimental measurements on the lower charge state suppression. The experimental arrangement was as follows.

The FTICR instrument is based on a 7 tesla solenoid magnet (Oxford instrument) and a vacuum system previously described [17]. The mass spectrometer incorporates an ESI ion interface with an electrodynamic ion funnel, an RF octopole for collisional focusing followed by RF quadrupoles for pre-selection, external accumulation and guiding ions into the cubic ICR cell. The vacuum system has six stages of differential pumping to drop gradually the pressure from atmospheric to high vacuum $\left(10^{-10}\right.$ torr) region of ICR trap.

The external accumulation interface consisted of an accumulation quadrupole having conductance limits located $1 \mathrm{~mm}$ from both ends of the quadrupole. The 49 $\mathrm{mm}$ long accumulation quadrupole has field radius 2.80 $\mathrm{mm}$ and rod (gold plated copper) diameters of $6.35 \mathrm{~mm}$. The conductance limits are gold plated stainless steel disks ( $30.5 \mathrm{~mm}$ o.d., $4 \mathrm{~mm}$ i.d., $0.5 \mathrm{~mm}$ width). The accumulation quadrupole was operated at a pressure 2 $\times 10^{-6}$ torr and was driven in RF-only mode (280 V $p-p$ at $1 \mathrm{MHz}$ ) by an in-house-built high-Q head controlled by a function generator and an RF amplifier. An Odyssey data station (Finnigan Corp., San Jose, CA) was used for the experimental sequence control.

During the ion accumulation event the DC potential of the front orifice was decreased to $2.5 \mathrm{~V}$, allowing ions to enter the quadrupole, while the exit orifice potential was set to $+5.6 \mathrm{~V}$. During the subsequent ion cooling event both orifice potentials were increased to $+7 \mathrm{~V}$, so that no additional ions could enter the quadrupole. (The $R F$ voltage on the ion optics prior to the accumulation quadrupole was also switched on during the ion accumulation event and switched off during the cooling and storing events.) Finally, during the ion storage event the front orifice potential was set at $+7 \mathrm{~V}$ and the exit orifice potential set to a lower potential to induce charge separation.

Peptide mixtures were dissolved in a water/methanol/acetic acid solution (49:49:2 vol\%) at concentration $10^{-6} \mathrm{M}$. All peptides used in this study (bradykinin, leucine enkephalin, hydra peptide fragment 7-11, substance P, fibrinopeptide A, DSIP, gamma-endorphin) were purchased from Sigma Chemicals (St. Louis, MO) and used without further purification. The solutions were infused into the ESI source at a flow rate of 20 $\mu \mathrm{L} / \mathrm{h}$ using a syringe pump (Harvard, South Natick, MA).

\section{Results}

Ions were accumulated in the accumulation quadrupole for $t_{\mathrm{acc}}=0.15$ or $0.3 \mathrm{~s}$. Then the ions were cooled for $2 \mathrm{~s}$. After the cooling event ions were stored during the storage time $t=0.01$ to $10 \mathrm{~s}$ with a reduced exit orifice

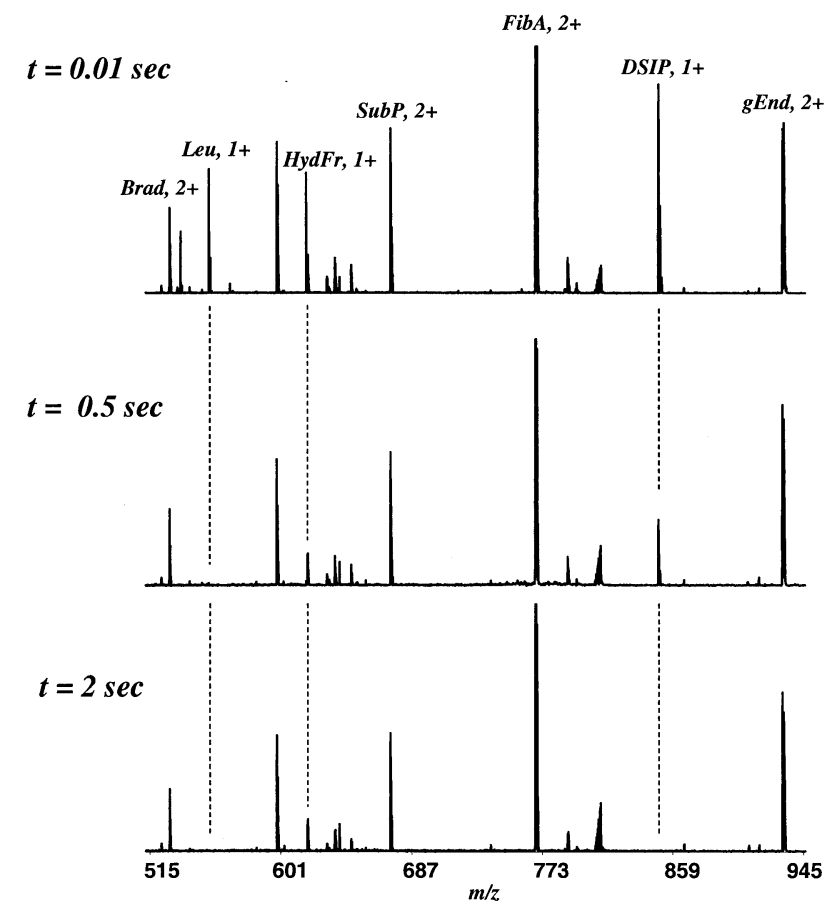

Figure 2. ESI mass spectra obtained from a $10^{-6} \mathrm{M}$ mixture of bradykinin (Brad), leucine enkephalin (Leu), hydra peptide fragment 7-11 (HydFr), substance P (subP), fibrinopeptide A (FibA), DSIP, gamma-endorphin (gEnd). Spectra were acquired for the storage time $t=0.01,0.5$, and $2 \mathrm{~s}$, for the exit orifice potential $V_{O R}$ $=0.7 \mathrm{~V}$.

potential $V_{O R}$. Then the ions were transferred into the ICR cell and mass spectra were acquired.

Ion storage for $V_{O R}$ above $1.5 \mathrm{~V}$ produced little effect on ion abundances, for storage times up to $10 \mathrm{~s}$. For an exit orifice potential in the range $0.7 \mathrm{~V}$ to $1.3 \mathrm{~V}$ singly charged ions were suppressed after the storage event. The storage time necessary to remove $1+$ ions sharply decreased for smaller voltages $V_{O R}$, as expected. Voltages lower than $0.5 \mathrm{~V}$ resulted in immediate loss of all trapped ions.

Figures 2 and 3 show mass spectra acquired for different storage times, $V_{O R}=0.7 \mathrm{~V}$, the accumulation time $t_{\text {acc }}=0.15 \mathrm{~s}$. Singly charged ions are suppressed for storage times $>\sim 0.5 \mathrm{~s}$, while $2+$ and $3+$ ions keep $>\sim 50 \%$ of the initial abundance. Figure 4 shows peak intensities versus storage time $t$, acquired for $V_{O R}=0.8$ $\mathrm{V}$. The decay rate observed for higher stored ion charge, $t_{\text {acc }}=0.3 \mathrm{~s}$, Figure $4 \mathrm{~b}$, is faster than one for $t_{\mathrm{acc}}=0.15 \mathrm{~s}$, Figure 4a. This indicates that the space charge is above the theoretical limit eq 9. Thus, we have chosen results obtained for the lower accumulation time, $t_{\mathrm{acc}}=0.15 \mathrm{~s}$, for comparison with theoretical predictions.

For these initial studies we disregard the effects of overfilling the trap that result in the faster ejection of highly charged species evident in Figures 3 and 4, and consequently reduced signal-to-noise ratios. The dynamic adjustment of the trapping voltage together with other approaches considered in the theoretical section may be used to ease this restriction. 


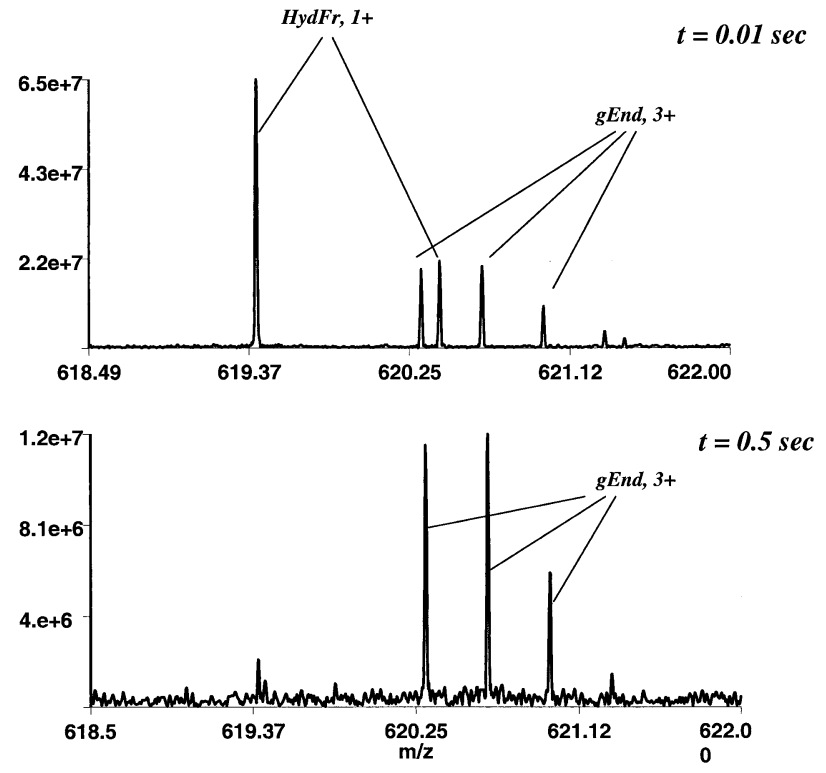

Figure 3. Segments of spectra in Figure 2 for storage times $t=$ $0.01 \mathrm{~s}$ and $0.5 \mathrm{~s}$, showing $1+$ and $3+$ charge state ions.

The primary parameter that defines the ion decay rate is the potential barrier height $U_{\text {trap }}$. In order to define this value we have performed $3 \mathrm{D}$ potential calculations for the configuration of electrodes used in the experimental setup. The potential profiles obtained for the exit orifice potential $V_{O R}=1.0 \mathrm{~V}$ are shown in Figure 5. DC offsets of the accumulation quadrupole, 0 $\mathrm{V}$, and the extraction quadruple, $-5 \mathrm{~V}$, correspond to potentials used in experiments. Potential profiles along the axis (z-coordinate) are shown for five different off-axis displacements in the $x$-direction, corresponding to the direction towards the quadrupole rod. One can see that for the orifice voltage of $1 \mathrm{~V}$ the potential barrier close to the axis, for $x=0.2$ and $0.7 \mathrm{~mm}$, is $\sim 0.2$ $\mathrm{V}$. The maximum potential for larger $x$-displacements increases and reaches $1 \mathrm{~V}$ for $x=2.2 \mathrm{~mm}$, larger than the orifice radius, $2 \mathrm{~mm}$.

Assuming that the ion cloud occupies a narrow radial region around the axis, we have used the axial potential profile, $x=0$, in order to estimate the potential barrier $U_{\text {trap }}$ as a function of the applied orifice voltage. This function is plotted in Figure 6. It can be seen that the potential barrier $U_{\text {trap }}=0.1 \mathrm{~V}$, used for estimations in Table 1, is set when the orifice potential is $V_{O R}=0.72$ $\mathrm{V}$.

Having the barrier height defined we can compare the observed kinetics of the ion decay with the theoretical relations, eq 6, 7. Figure 7 shows the peak intensity decay kinetics for different orifice voltages, for $1+$ leucine enkephalin ion, $m / z=556$. Experimental results (symbols) are plotted together with theoretical decay curves. This is a direct comparison of the theory and experiment, involving no free (or adjustable) parameters. The experimental decay rate roughly follows theoretical predictions. The decay time for lower potential barriers, for $V_{O R}=0.7 \mathrm{~V}$ and $0.8 \mathrm{~V}$, is noticeably larger
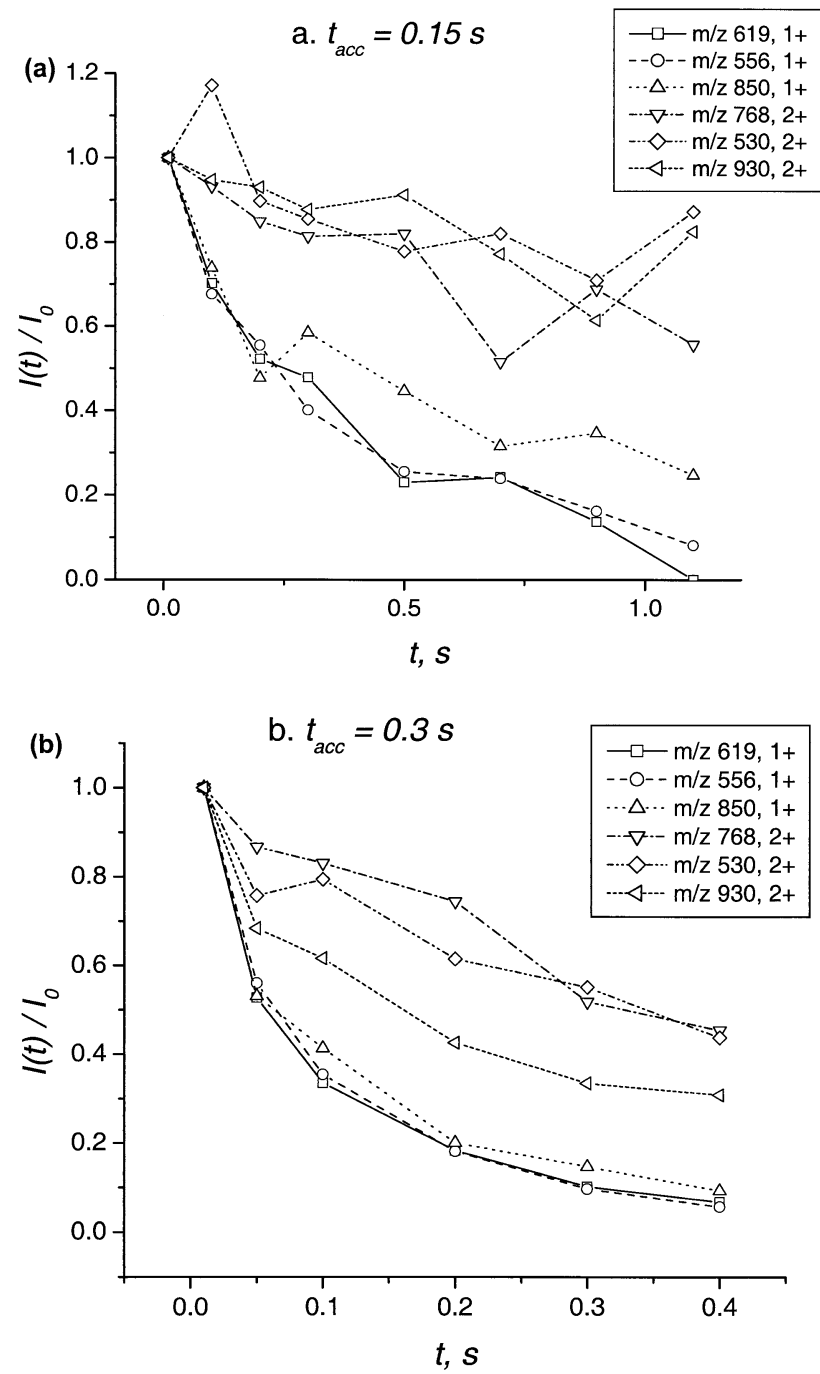

Figure 4. Relative peak intensities versus storage time for selected $1+$ and $2+$ ions, for the exit orifice potential $V_{O R}=0.8 \mathrm{~V}$. The initial amount of stored ion charge corresponds to the accumulation time $t_{\mathrm{acc}}=0.15 \mathrm{~s} \mathrm{(a)}$ and $0.30 \mathrm{~s}(\mathbf{b})$.

than theoretical one. We attribute this discrepancy as likely due to an insufficient bath gas pressure in the accumulation quadrupole. As discussed above, in order to fulfill the quasi-equilibrium conditions, the thermal relaxation time $\tau$, eq 1 , must be smaller than the characteristic decay time $\tau_{i}$. The bath gas pressure used, $2 \times 10^{-6}$ torr, corresponds to the thermal relaxation exponential time $\tau \sim 0.4 \mathrm{~s}$, which is of the same order of magnitude as the decay time $\tau_{i}<\sim 0.5 \mathrm{~s}$ for the two lower trapping voltages. One can assume that the loss of the high kinetic energy ions, corresponding to the high energy tail of the thermal distribution, can not be fully compensated by the ion-neutral collisions under such conditions, resulting in a slower than expected decay rate. For higher trapping voltages, 0.9 and $1.0 \mathrm{~V}$, the decay time $\tau_{i}>\sim 1 \mathrm{~s}$ is still comparable to the thermal relaxation time, but the observed kinetics are quite close to the theoretical. This observation suggests that relatively low bath gas pressures, $\sim 10^{-6}$ torr, can 


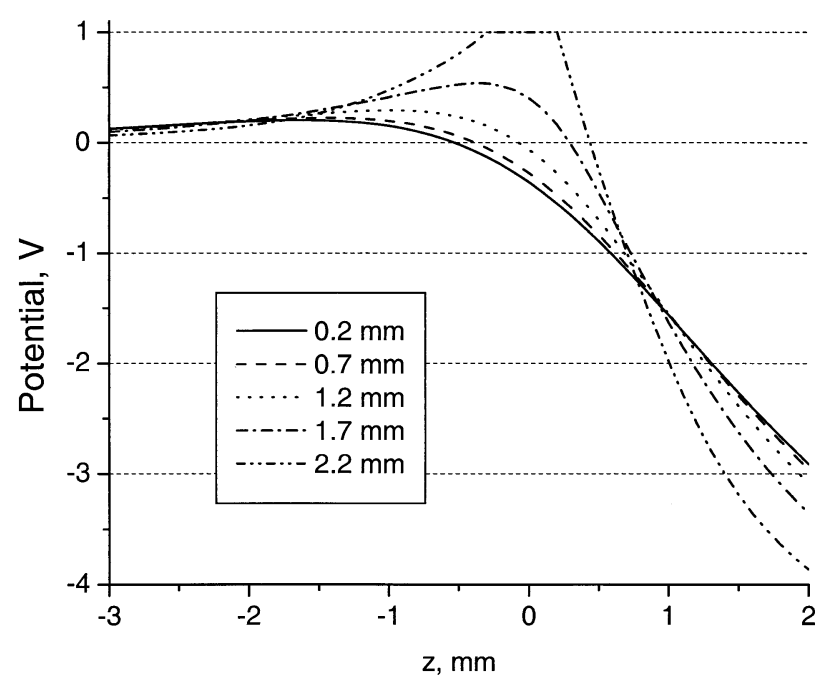

Figure 5. Potential profiles calculated for the electrode configuration used for experimental measurements. The exit orifice potential $V_{O R}=1.0 \mathrm{~V}$. Off-axis displacement $x=0.2,0.7,1.2,1.7$, and $2.2 \mathrm{~mm}$. The orifice $4 \mathrm{~mm}$ i.d., $0.5 \mathrm{~mm}$ thick, is positioned at $z=$ $-0.2 \mathrm{~mm}$. Two RF quadrupoles with $R_{0}=2.8 \mathrm{~mm}$ are positioned at $z=-1.2 \mathrm{~mm}$ and $z=1.3 \mathrm{~mm}$. The accumulation quadrupole has DC offset $0 \mathrm{~V}$, and the extraction quadrupole is at $-5 \mathrm{~V}$.

be used for the low charge state suppression, provided that the barrier is adjusted to the decay times of $>\sim 1 \mathrm{~s}$.

\section{Conclusions}

The present experimental results agree well with the exponential decay relation, eq 6 , developed for conditions of sufficiently low space charge and high DC barrier, and where the characteristic decay time is larger than the thermal relaxation time. Thus, the physical model considered in the theoretical section appears to

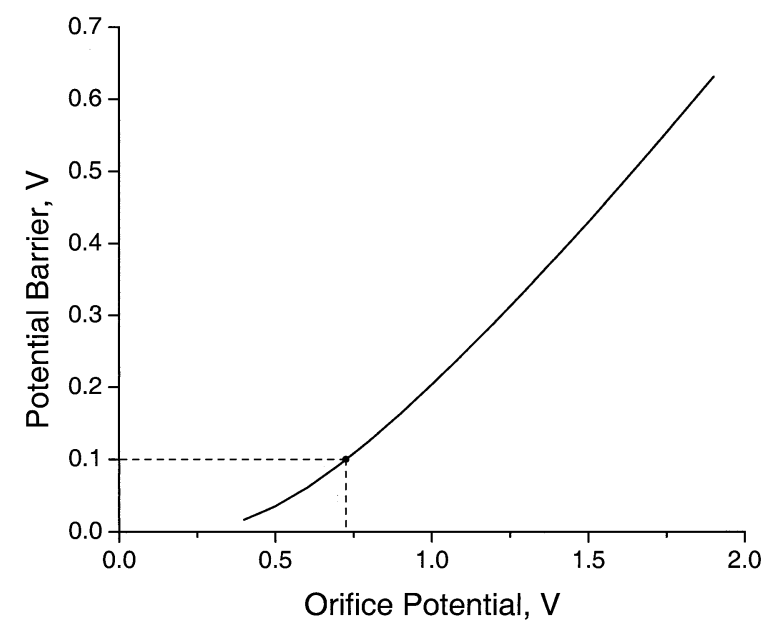

Figure 6. The potential barrier, calculated as the axial DC potetial maximum, vs. the orifice potential. Potential computations have been performed for dimensions used in experiment, see caption for Figure 5. The selected point shows that the axial potential barrier of $0.1 \mathrm{~V}$ corresponds to the orifice potential of $0.72 \mathrm{~V}$.

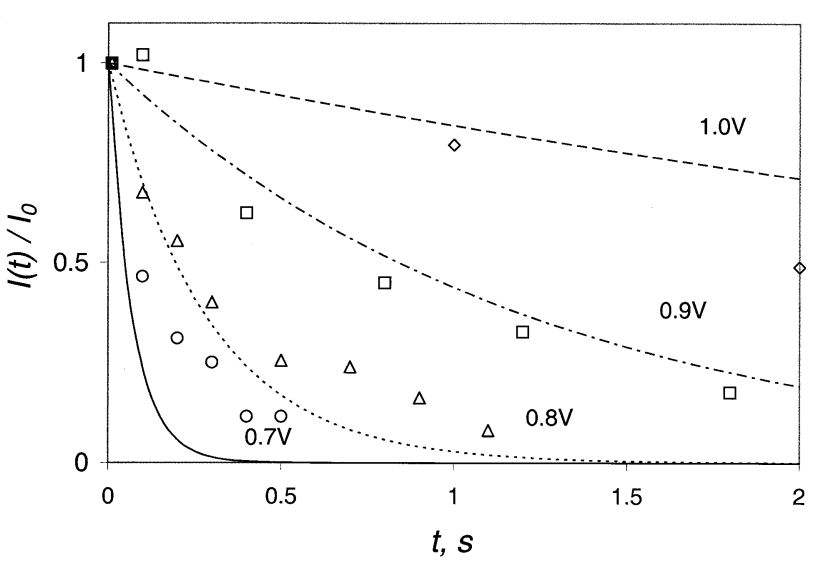

Figure 7. Relative peak intensity for leucine enkephalin 1+ ion, $\mathrm{m} / \mathrm{z}=556$, versus storage time $t$, for exit orifice voltages $0.7,0.8$, 0.9, and 1.0 V. Experimental data: Symbols. Curves show the theoretical kinetics, eq 6, 7, using potential barrier values estimated by means of $3 \mathrm{D}$ potential calculations.

provide an adequate description for the observed phenomenon.

Further studies should address the practical utility of the lower charge state suppression, e.g., for removing the singly charged chemical noise due to solvent and other contaminants in LC-MS or ampholytes in on-line CIEF separations. In order to overcome the restriction on the stored charge quantity, eq 9, one can use a time-dependent trapping potential $V(t)$, rather than the constant low potential used in the present study. A more detailed characterization of the efficiency of the charge state separation process for various charge state ions, for an optimal pressure regime and optimal RF parameters of the ion accumulation multipole appears worthwhile.

\section{Acknowledgments}

The authors thank Dr. K. Tang for helpful discussions and Drs. B. Bogdanov and S. Ahn for assistance in experimental measurements. Portions of this research were supported by the U.S. Department of Energy, Office of Biological and Environmental Research. The Pacific Northwest National Laboratory is operated by Battelle Memorial Institute for the U.S. Department of Energy through contract DE-AC06-76RLO 1830.

\section{References}

1. Wollnik, H. Ion Optics in Mass Spectrometers. J. Mass Spectrom. 1999, 34, 991-1006.

2. Douglas, D. J. Applications of Collision Dynamics in Quadrupole Mass Spectrometry. J. Am. Soc. Mass Spectrom. 1998, 9, 101-113.

3. Douglas, D. J.; French, J. B. Collisional Focusing Effects in Radio Frequency Quadrupoles. J. Am. Soc. Mass Spectrom. 1992, 3, 398-408.

4. Tolmachev, A. V.; Chernushevich, I. V.; Dodonov, A. F.; Standing, K. G. A Collisional Focusing Ion Guide for Coupling an Atmospheric Pressure Ion Source to a Mass Spectrometer. Nucl. Instr. Meth. Phys. Res. B. 1997, 124, 112-119.

5. Tolmachev, A. V.; Udseth, H. R.; Smith, R. D. The Charge Capacity Limitations of Radio Frequency Ion Guides in Their 
Use for Improved Ion Accumulation and Trapping in Mass Spectrometry. Anal. Chem. 2000, 72, 970-978.

6. Tolmachev, A. V.; Udseth, H. R.; Smith, R. D. Radial Stratification of Ions as a Function of Mass to Charge Ratio in Collisional Cooling Radio Frequency Multipoles Used as Ion Guides or Ion Traps. Rapid Commun. Mass Spectrom. 2000, 14, 1907-1913.

7. Tolmachev, A. V.; Udseth, H. R.; Smith, R. D. Modeling the Ion Density Distribution in Collisional Cooling RF Multipole Ion Guides. Int. J. Mass Spectrom. 2003, 222, 155-174.

8. Senko, M. W.; Hendrickson, C. L.; Emmett, M. R.; Shi, S. D.; Marshall, A. G. External Accumulation of Ions for Enhanced Electrospray Ionization Fourier Transform Ion Cyclotron Resonance Mass Spectrometry. J. Am. Soc. Mass Spectrom. 1997, 8, 970-976.

9. Campbell, J. M.; Collings, B. A.; Douglas, D. J. A New Linear Ion Trap Time-of-Flight System with Tandem Mass Spectrometry Capabilities. Rapid Commun. Mass Spectrom. 1998, 12, 1463-1474.

10. Sannes-Lowery, K.; Griffey, R. H.; Kruppa, G. H.; Speir, J. P.; Hofstadler, S. A. Multipole Storage Assisted Dissociation, a Novel In-Source Dissociation Technique for Electrospray Ionization Generated Ions. Rapid Commun. Mass Spectrom. 1998, 12, 1957-1961.

11. Belov, M. E.; Nikolaev, E. N.; Anderson, G. A.; Udseth, H. R.; Conrads, T. P.; Veenstra, T. D.; Masselon, C. D.; Gorshkov, M. V.; Smith, R. D. Design and Performance of an ESI Interface for Selective External Ion Accumulation Coupled to a Fourier
Transform Ion Cyclotron Mass Spectrometer. Anal. Chem. 2001, 73, 253-261.

12. Harkewicz, R.; Belov, M. E.; Anderson, G. A.; Paša-Tolic, L.; Masselon, C. D.; Prior, D. C.; Udseth, H. R.; Smith, R. D. ESI-FTICR Mass Spectrometry Employing Data-Dependent External Ion Selection and Accumulation. J. Am. Soc. Mass Spectrom. 2002, 13, 144-154.

13. Chernushevich, I. V.; Fell, L. M.; Bloomfield, N.; Loboda A. V.; Shevchenko, A. A. Charge Separation for Protein Applications on a QqTOF Mass Spectrometer. Proceedings of the 50th ASMS Conference on Mass Spectrometry and Allied Topics; Orlando, FL, June, 2002;

14. Keshishian, H.; Addona, T.; Clauser, K.; Yves LeBlanc, J. C.; Chu, I. K.; Carr, S. A. MS and MS/MS Performance of a New Triple Quadrupole-Linear Ion Trap Mass Spectrometer for Proteomics. Proceedings of the 50th ASMS Conference on Mass Spectrometry and Allied Topics; Orlando, FL, June, 2002;

15. Chernushevich, I. V.; Fell, L. M.; Bloomfield, N.; Metalnikov, P. S.; Loboda, A. V. Charge State Separation for Protein Applications Using a Quadrupole Time-of-Flight Mass Spectrometer. Rapid Commun. Mass Spectrom. 2003, 17, 1416-1424.

16. Gerlich, D. In State Selected and State-to-State Ion-Molecule Reaction Dynamics. Part 1. Experiment, Vol. LXXXII; Ng, C. Y.; Baer, M., Eds.; Wiley: New York, 1992; pp 1-176.

17. Winger, B.; Light-Wahl, K.; Ogorzalek-Loo, R.; Udseth, H. R.; Smith, R. D. Observation and Implications of High Mass-toCharge Ratio Ions from Electrospray Ionization Mass Spectrometry. J. Am. Soc. Mass Spectrom. 1993, 4, 536-545. 